OPEN

SUBJECT AREAS:

TUBERCULOSIS

HEALTH SCIENCES

Received

9 December 2013

Accepted

3 February 2014

Published

28 February 2014

Correspondence and requests for materials should be addressed to

D.R. (didier.raoult@ gmail.com) or M.D. (michel.drancourt@ univ-amu.fr)

\title{
Dramatic reduction of culture time of Mycobacterium tuberculosis
}

\author{
Ramzi Ghodbane, Didier Raoult \& Michel Drancourt
}

Aix Marseille Université, URMITE UMR CNRS 7278, IRD 198, Inserm 1095, 13005, Marseille, France.

Mycobacterium tuberculosis culture, a critical technique for routine diagnosis of tuberculosis, takes more than two weeks. Here, step-by-step improvements in the protocol including a new medium, microaerophlic atmosphere or ascorbic-acid supplement and autofluorescence detection dramatically shortened this delay. In the best case, primary culture and rifampicin susceptibility testing were achieved in 72 hours when specimens were inoculated directly on the medium supplemented by antibiotic at the beginning of the culture.

M ycobacterium tuberculosis is the causative agent of tuberculosis, which results in 1.3 million deaths and nine million new cases each year ${ }^{1}$. A major problem when dealing with tuberculosis has been a difficulty in diagnosis due to slow growth of mycobacterial cultures, which subsequently explains the slow process of evaluating the susceptibility of this microorganism to antibiotics. Using current tools, a primary culture is obtained in two to four weeks on average, and antibiotic susceptibility is determined after an additional two to four weeks $s^{2,3}$. Therefore, four to eight weeks are needed to obtain an isolate and determine its susceptibility to antibiotics. Molecular methods have been proposed to replace this protocol ${ }^{4}$. However, new techniques are available to optimize the culture system as recently highlighted in the phenomenon of the rebirth of culture ${ }^{5}$. A first step is the optimization of the culture medium ${ }^{6}$. A second step is the strict control of oxygen tension, a parameter that has not been widely evaluated in $M$. tuberculosis cultures but may potentially help in optimizing the cultures. A third step is the early detection of growth ${ }^{7}$. A fourth step is the use of matrix-assisted laser desorption/ionization time-of-flight mass spectrometry (MALDI-TOF-MS) for identification ${ }^{8}$. Finally, the development of rapid antibiotic susceptibility testing can allow a reasonable timeframe to detect resistance. We experimentally investigated each of these steps to obtain the optimal culture conditions for antibiotic susceptibility testing and obtain results in less than seven days.

To optimize the culture medium, we started with the blood medium previously reported ${ }^{6}$. By chance, we found that the egg lecithin, which is used as an antidote to the chlorhexidine that is used to decontaminate specimens ${ }^{9}$, had a growth-promoting effect. In addition, we tested different concentrations of the reagents used to make the medium and varied the $\mathrm{pH}$ (Supplementary Fig. 1, Supplementary Table 1, Supplementary Table 2). We were able to develop a medium that promoted additional growth in both liquid medium and agar. Using this medium, we obtained microbial growth in $8.4 \pm 3$ days for 20 clinical sputum specimens (primary cultures) (Fig. 1) and in $5 \pm$ 1 days for the reference M. tuberculosis $\mathrm{H} 37 \mathrm{Rv}$ strain and 55 clinical isolates (subcultures) (Supplementary Fig. 2). These results were compared with the results obtained with the same specimens using the routine automated liquid medium, Bactec ${ }^{\mathrm{TM}}$ (Becton Dickinson); here, microbial growth occurred in $12.55 \pm 4.6$ days (primary cultures) and in $6.6 \pm 2$ days (subcultures) $(P<0.05)$.

Next, we evaluated the role of the atmosphere on growth in our solid medium, as it has been found to be a critical factor for intracellular bacterial growth ${ }^{10}$. We observed improved growth under a microaerophilic atmosphere compared to normal oxygen tension. Primary cultures from the clinical specimens were detected one day earlier under microaerophilic conditions than under normal atmosphere. The culture time decreased from $8.4 \pm$ 3 to $7.4 \pm 2.4$ days for the primary culture and from $5 \pm 1$ to $4.4 \pm 2$ days for the subculture $(P<0.05)$. It therefore appears that a microaerophilic atmosphere (not yet reported in the literature) is a critical factor in reducing the culture time of $M$. tuberculosis. At this stage, by combining our medium and the microaerophilic atmosphere, we decreased the culture time by approximately $40 \%$ (from 12.55 to 7.4 days for the primary culture and from 6.6 to 4.4 days for the subculture).

The detection of microcolonies has been found to save time for diagnosis ${ }^{7}$. We compared the size of 10 detectable colonies using the naked eye, a binocular microscope and autofluorescence and evaluated the number of mycobacteria per colony by cytometer (Fig. 2a). Naked eye detection observed colonies of $943 \pm 51 \mu \mathrm{m}$ in diameter containing $6.4 \pm 3.5 \times 10^{5}$ mycobacteria; by binocular microscopy, we detected colonies of 


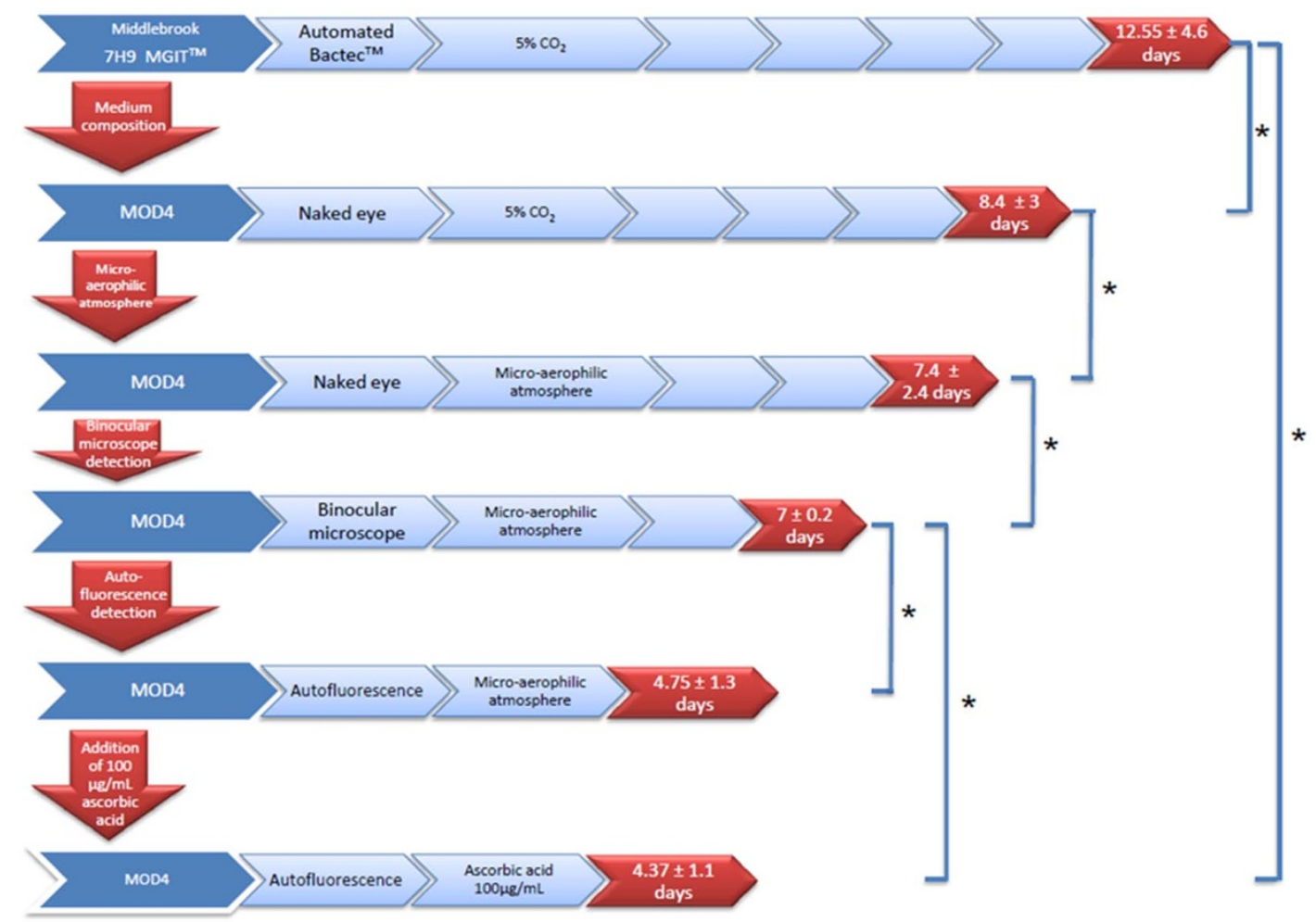

Figure 1 Comparison of different protocols for the culture of M. tuberculosis. A total of 20 sputum specimens were cultured using different protocols, and the time of detection of M. tuberculosis in primary cultures was measured. Step-by-step modifications included medium composition, atmospheric control and ultimate detection of autofluorescent microcolonies. ${ }^{*} P<0.05$, Student's $t$ test.

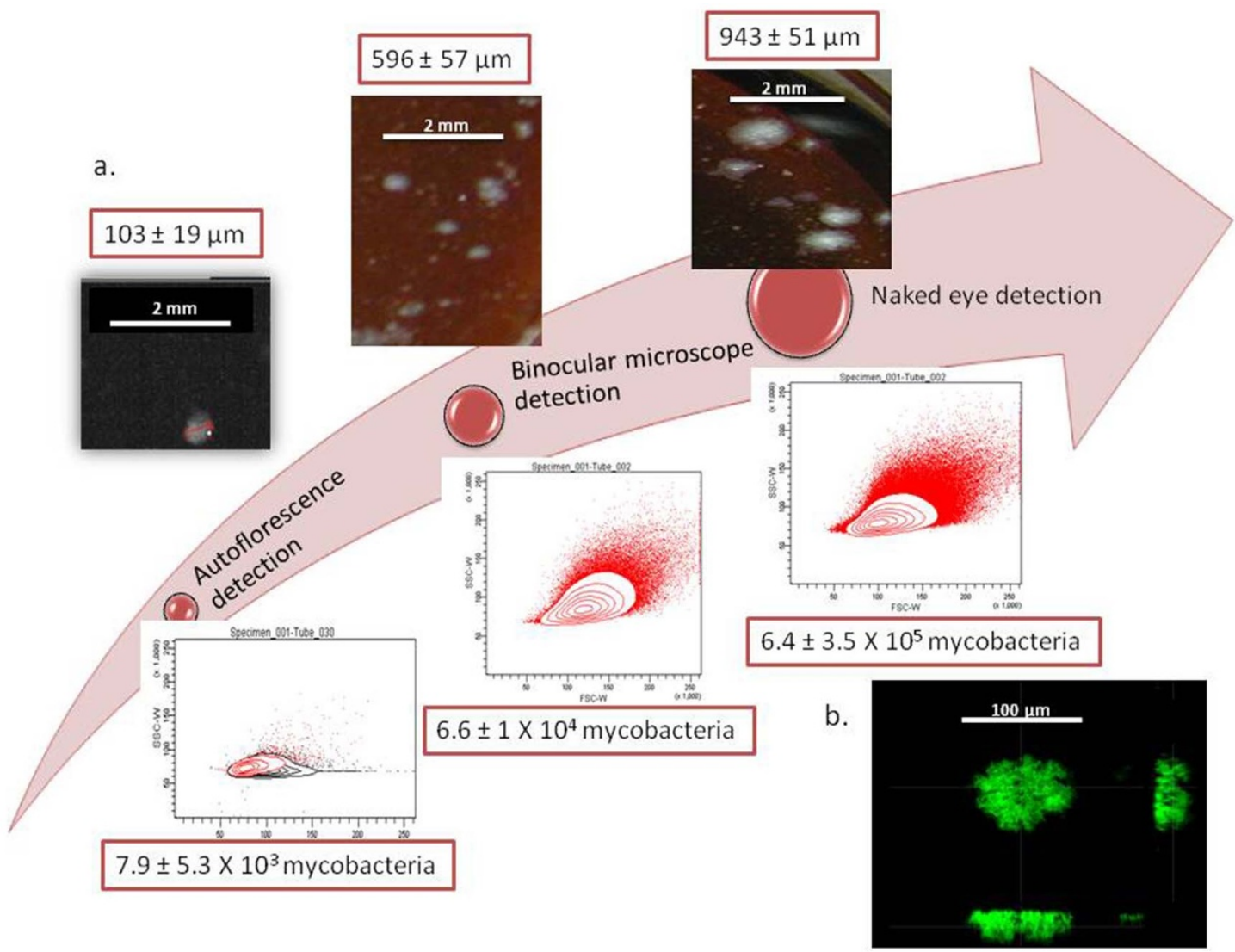

Figure $2 \mid$ Comparison of the three methods to detect $M$. tuberculosis colonies. (a), Size and mycobacterial load when detecting microcolonies by autofluorescence, by binocular microscope and by naked eye. (b), Confocal microscopic image of a 90- $\mu \mathrm{m}$ autofluorescent microcolony of $M$. tuberculosis grown on MOD4 medium. 
$596 \pm 57 \mu \mathrm{m}$ in diameter containing $6.6 \pm 1 \times 10^{4}$ mycobacteria; by autofluorescence, we detected microcolonies of $103 \pm 19 \mu \mathrm{m}$ in diameter containing $7.9 \pm 5.3 \times 10^{3}$ mycobacteria. We used confocal microscopy to visualize the form and size of the autofluorescent microcolonies. These analyses showed the rough hemispheric form and structure of a $90-\mu \mathrm{m}$ microcolony (Fig. 2b). Next, microcolonies were collected and prepared for MALDI-TOF-MS analysis using a previously described protocol ${ }^{8}$ (Supplementary Fig. 3a \& b), and we found that $10^{4}$ mycobacteria (i.e., a microcolony of $496 \pm 57 \mu \mathrm{m}$ in size) could be identified by this technique (Supplementary Fig. 3c).

Combining an optimal medium, optimized incubation conditions and powerful detection tools, we obtained primo-isolation from clinical sputum in $4.75 \pm 1.3$ days compared to $12.55 \pm 4.6$ days with routine protocol using Bactec MGIT $(P<0.05)$ (Fig. 1). In order to further simplify the process, we tested whether the addition of the antioxidant ascorbic acid would allow growth under normal oxygen tension. Primary culture from the clinical specimens grew in $4.26 \pm$ 1.3 days in microaerophilic atmosphere; and in $4.37 \pm 1.1$ days in our solid medium complemented with $100 \mathrm{mg} / \mathrm{L}$ ascorbic acid and under normal atmosphere. These data proved that it was possible to resume microaerophilic atmosphere with the addition of ascorbic acid at a non-toxic final concentration ${ }^{11}$ (Fig. 1).

With respect to testing for antibiotic susceptibility, we developed a strategy in which one six-well microplate was inoculated with a critical concentration of the four first-line antituberculous antibiotics and an antibiotic-free well used as a positive control for growth. The results were determined by the presence or absence of fluorescent microcolonies every 12 hours of incubation for $23 \mathrm{M}$. tuberculosis clinical isolates, including one rifampin-resistant isolate and three $\mathrm{XDR}$ isolates. For the positive control well the average time for growth decreased from $6.6 \pm 2$ days to $2.43 \pm 0.82$ days for the three XDR strains. The susceptibility testing time decreased from $6.6 \pm 2$ days in automated Bactec $^{\mathrm{TM}}$ liquid medium to $2.43 \pm 0.82$ days with our protocol $(P<0.05)$.

To evaluate the efficacy of the different modifications (medium, microaerophilic conditions, autofluorescent microcolony detection, MALDI-TOF-MS and antibiotic susceptibility testing), we tested five sputum specimens. To determine inoculum size, we quantified the mycobacteria by Ziehl staining, by FacScan and by quantitative PCR. We observed a correlation between the different counting methods. The specimens were tested both fresh (kept at $4{ }^{\circ} \mathrm{C}$ ) and after freezing at $-80^{\circ} \mathrm{C}$. The primary culture time was $102 \pm 36$ hours for fresh specimens and $156 \pm 53$ hours for frozen specimens $(P<0.05)$. We also found a negative correlation between bacterial load and growth time $(\mathrm{r}=-0.93)$; for example, specimens containing $\geq 10^{6}$ mycobacteria yielded colonies within $72 \mathrm{~h}$, those containing $10^{5}$ mycobacteria yielded colonies between 96 and $110 \mathrm{~h}$ and those containing $<10^{5}$ mycobacteria yielded colonies in $160 \mathrm{~h}$ (Supplementary Fig. 4). Antibiotic susceptibility testing involving three highly resistant mycobacteria (XDR), and two susceptible mycobacteria strains were obtained in $144 \pm 28$ hours, with the shortest time from inoculation of the primary culture to susceptibility testing in the two susceptible strains being 72 and 36 hours.

In clinical microbiology, culture methods have been highly underestimated over the last 20 years, and only a few types of media have been optimized. Important efforts have been directed towards tuberculosis surveillance, but these efforts have been largely focused on techniques for early detection of metabolic activity in media by robots or by molecular detection ${ }^{4}$. New growth strategies incorporating cell extracts have also been developed in our laboratory, including methods for intracellular, fastidious bacteria such as Mycobacterium bovis $^{10}$. In parallel, environmental microbiologists have developed new strategies that have allowed for the enrichment of a singular type of culture technique. Here, we applied these principles by providing axenic cultures in conditions mimicking the intracellular environment, $M$. tuberculosis being a facultative intracellular organism infecting amoeba ${ }^{12}$, macrophages ${ }^{13}$ and adipocytes ${ }^{14}$. We found that microaerophilia (about $12 \mu \mathrm{moL}$ oxygen) and scavangering oxygen by ascorbic acid significantly stimulated the axenic growth of $M$. tuberculosis, while the intracellular concentration of oxygen is of $<15 \mu \mathrm{moL}^{15}$. Blood, serum and glucose specifically added in our medium are diffusing into tissues and cells infected by $M$. tuberculosis. Also, lecithin constituting our medium is a key component of cell membranes which are degraded by lipolytic enzymes to provide $M$. tuberculosis with essential lipids ${ }^{16}$. These observations provide a rationale for empirical treatments of pulmonary tuberculosis used in the pre-antituberculous area. Oxygen therapy ${ }^{17}$ probably worked by increasing oxygen concentration in infected lungs, limiting the growth of $M$. tuberculosis ${ }^{18}$. Surgical collapse therapies reduced the access of infected lung tissues and $M$. tuberculosis to blood ${ }^{19}$. Indeed, there is a renewed interest for these older therapeutic options, in the current area of XDR tuberculosis ${ }^{19}$.

Our findings showed that M. tuberculosis culture could progress quickly from isolating clinical specimens to antibiotic testing using a modification of current methods. In fact, using our methodology, we were able to isolate XDR $M$. tuberculosis from one sputum sample in 72 hours and determine its resistance to rifampicin in additional 36 hours. Moreover, when we tested antibiotic susceptibility in parallel with primary inoculation in ten sputum specimens, we were able to reduce the diagnosis time to 72 hours in two Ziehl-positive patients including one XDR M. tuberculosis. Any antibiotic could be tested by using this versatile assay for the discovery of new resistance. It should be noted that this culture strategy can be adapted for use in resource-poor countries. Such an optimization of the culture of M. tuberculosis also serves as a proof-of-concept for the rebirth of microbial culture and the need to reinvest in this field.

\section{Methods}

Mycobacterial strains and clinical specimens. The M. tuberculosis $\mathrm{H} 37 \mathrm{Rv}$ CIP104475T strain and 55 M. tuberculosis clinical isolates obtained during the routine activity of the Mycobacteria Reference Laboratory, Institut Méditerranée Infection, Marseille, France were used in this study. All manipulations were performed in a biosafety level 3 laboratory using all safety precautions. All isolates were re-suspended rigorously in phosphate-buffered saline (PBS) (Biomérieux, La Balme-les-Grottes, France) with 5-mm glass beads (Sigma, Saint Quentin Fallavier, France), and the suspension was diluted to $10^{8}$ colony-forming units (cfu) $/ \mathrm{mL}$ as measured by optical density at $580 \mathrm{~nm}$ (Cell Density Meter; Fisher Scientific, Illkirch, France). Twenty sputum specimens, including 10 specimens exhibiting acid-fast bacilli upon microscopic observation after Ziehl-Armand staining, were also prospectively included in this study. Prior to inoculation, all specimens were decontaminated using the NALC-NaOH method with the BD MycoPrep ${ }^{\text {TM }}$ Specimen Digestion/ Decontamination Kit (Becton Dickinson, Pont de Claix, France). An equal volume of $4 \% \mathrm{NaOH}$ and the sputum specimen were mixed together in a $50-\mathrm{mL}$ tube. Next, the mixture was homogenized and incubated at room temperature for $15 \mathrm{~min}$. After incubation, the $\mathrm{NaOH}$ was neutralized by adding PBS, and the suspension was centrifuged for $15 \mathrm{~min}$ at $1,000 \times$ g. A $100-\mu \mathrm{L}$ aliquot of each decontaminated sputum specimen was inoculated on plates and incubated at $37^{\circ} \mathrm{C}$ for up to 20 days. In each culture batch, negative culture controls were processed in parallel to detect contamination at the ratio of one negative control to four tested specimens.

MOD4 formulation optimization. To optimize the blood-based agar medium, 12 different compounds were assessed for their effect on the growth of the test strain, $M$. tuberculosis H37Rv, versus the growth of this strain on the reference Middlebrook 7H10 medium (Becton Dickinson) used as the gold standard. The following compounds were tested at varying concentrations: selenium, egg lecithin, yeast extract (Sigma), sheep blood, chicken blood, horse blood (Biomérieux), pancreatic digest of casein (Sigma), glucose, mycobactine J (Symbiotic Europe, Lyon, France), Fastidious Organism Supplement (Becton Dickinson), methyl- $\beta$-cyclodextrin (Sigma) and heatinactivated fetal bovine serum (Gibco Life Technologies, Saint Aubin, France). To optimize the combination of concentrations for compounds with positive effects, we used a matrix to cross all compounds and concentrations (Supplementary Fig. 1).

For media preparation, we weighed $19 \mathrm{~g}$ of Middlebrook $7 \mathrm{H} 10$ powder, one gram of yeast extract, two grams of glucose and one gram of pancreatic digest of casein and added $2.5 \mathrm{~mL}$ of glycerol and Tween 80 (Sigma). All compounds were dissolved in $623 \mathrm{~mL}$ of distilled water, and the mixture was autoclaved at $121^{\circ} \mathrm{C}$ for $20 \mathrm{~min}$. A 50 $\mathrm{mL}$ volume of antibiotic solution (1 g of vancomycin, 6,000 units of polymyxin B, $600 \mathrm{mg}$ of amphotericin B, 2,400 mg of nalidixic acid, $600 \mathrm{mg}$ of trimethoprim and $600 \mathrm{mg}$ of azlocillin), $100 \mathrm{~mL}$ of OADC (oleic acid albumin, dextrose, catalase growth supplement (Becton Dickinson), $50 \mathrm{~mL}$ of blood, $50 \mathrm{~mL}$ of heat-inactivated fetal bovine serum and $20 \mathrm{~mL}$ of fastidious organism supplement were added after the medium was cooled to $55^{\circ} \mathrm{C}$. The medium was poured onto Petri dishes (Dominique 
Dutscher, Brumath, France), and Middlebrook 7H10 was prepared according to Becton Dickinson's protocol. All experiments were repeated four times, and plates inoculated with sterile PBS were used as negative controls.

Culture conditions and growth monitoring. Several environmental conditions for $M$. tuberculosis growth were tested, including temperature $\left(25^{\circ} \mathrm{C}, 30^{\circ} \mathrm{C}, 37^{\circ} \mathrm{C}\right.$ and $\left.40^{\circ} \mathrm{C}\right)$, $\mathrm{pH}(5,6,7,8$ and 9$)$ and atmosphere (aerobic, $5 \% \mathrm{CO}_{2}$, microaerophilic with $2.5-5 \%$ $\mathrm{O}_{2}, 2.5-5 \% \mathrm{O}_{2}$ plus $5 \% \mathrm{CO}_{2}$ and anaerobic). The $\mathrm{CO}_{2}$ atmosphere was obtained via a GasPakTM EZ $\mathrm{CO}_{2}$ Gas Generating Pouch System (Becton Dickinson), and the microaerophilic condition was generated using a CampyGen ${ }^{\mathrm{TM}}$ pack (Oxoid, Dardilly, France). Fifteen $M$. tuberculosis isolates comprising M. tuberculosis $\mathrm{H} 37 \mathrm{Rv}$ as the reference strain and fourteen clinical isolates were used to compare the culture conditions (Supplementary Table 2). A 100- $\mu \mathrm{L}$ aliquot of each M. tuberculosis isolate $\left(10^{8} \mathrm{cfu} / \mathrm{mL}\right)$ was inoculated in both the Middlebrook $7 \mathrm{H} 10$ reference medium (Becton Dickinson) and the medium being developed. Microcolonies were detected by observing $M$. tuberculosis autofluorescence at $12.5 \mathrm{X}$ magnification between $450 \mathrm{~nm}$ and $550 \mathrm{~nm}^{20}$ using a binocular microscope with fluorescence detection and a monochrome camera (MZ10 F stereomicroscope, Leica Microsystems, Nanterre, France) equipped with a GFP filter. Detection of microcolonies was initiated at 24 hours post-inoculation and was performed every 12 hours until day 20 post-inoculation; detection was performed using microscopy with autofluorescence, by loop and by the naked eye. We also counted the number of mycobacteria by auto-fluorescence using a BD LSRFortessaTM cell analyzer with BD FACSDivaTM software (Becton Dickinson); this method enabled us to detect $80-200-\mu \mathrm{m}, 500-700-\mu \mathrm{m}$ and $900-1,000-\mu \mathrm{m}$ colonies. As controls, colonies of Staphylococcus aureus, Pseudomonas aeruginosa and Candida albicans clinical isolates were observed under the same conditions. For antibiotic susceptibility testing, $23 \mathrm{M}$. tuberculosis isolates including one rifampicin-resistant isolate and three XDR isolates were tested for rifampicin (Sigma) incorporated into the medium at $2 \mathrm{mg} / \mathrm{L}$, isoniazid (Sigma) at $0.2 \mathrm{mg} / \mathrm{L}$, ethambutol (Sigma) at $5 \mathrm{mg} / \mathrm{L}$ and streptomycin (Sigma) at $7 \mathrm{mg} / \mathrm{L}$. All experiments were repeated five times.

Colony identification. To determine the most useful method of identifying microcolonies, we evaluated several techniques, including Ziehl staining, MALDITOF-MS and the GeneXpert ${ }^{\circledR}$ System (Cepheid, Maurens-Scopont, France). Ziehl staining was performed with an automatic AT-2000Z stainer (Biocentric, Bandol, France). For MALDI-TOF-MS identification, microcolonies were collected and prepared using the previously described protocol8 and then applied onto a MALDITOF-MS 96 Microflex target (Bruker Daltonic, Wissenbourg, France). We used the GeneXpert ${ }^{\circledR}$ MTB/RIF assay according to the supplier's recommendations (Cepheid).

Confocal microscopic imaging. To observe confocal images of microcolonies, microcolonies of approximately $100 \mu \mathrm{m}$ were carefully removed and placed on microscope slides. The microcolonies were covered with $70 \%$ ethanol for 1 hour to inactivate the organisms. The slides were then observed with a LEICA SP5 twophoton resonant scanner confocal microscope (Leica Microsystems) with excitation lasers at $405 \mathrm{~nm}, 488 \mathrm{~nm}, 543 \mathrm{~nm}$ and $633 \mathrm{~nm}$ and a two-photon system at $1,030 \mathrm{~nm}$. Images were taken at $100 \mathrm{X}$ magnification with excitation at $405 \mathrm{~nm}$, and the data were analyzed with the Autoquant TM deconvolution algorithm using LAS AF software (Leica Microsystems).

Clinical specimens. To identify other optimal conditions for culture, we determined the effect of freezing the clinical specimens on culture time. To test this parameter, we cultured five fresh specimens that were Ziehl-positive and the same five specimens after they were frozen. We also quantified the amount of $M$. tuberculosis in each specimen by quantitative real-time PCR. The 151-bp Internal Transcribed Spacer (ITS) of M. tuberculosis was targeted using the pUC57 plasmid containing the ITS sequence of $M$. tuberculosis (Eurogentec, Angers, France). We used standard solutions containing $M$. tuberculosis at concentrations ranging from $10^{3}$ to $10^{7} \mathrm{cfu} /$ $\mathrm{mL}$. The real-time PCR was performed with a Bio-Rad CFX96 RT C1000 (Bio-Rad, Marnes-la-Coquette, France). The specimens were placed on MOD4 medium and incubated according to the protocol described above. Colony monitoring was performed every $12 \mathrm{~h}$ using the autofluorescence method, and detected microcolonies were subcultured on MOD4 and MOD4 supplemented with rifampicin at $2 \mathrm{mg} / \mathrm{L}$ (Sigma). Also, the effect of $100 \mathrm{mg} / \mathrm{L}$ ascorbic acid (VWR international, Fontenay-sous-Bois, France) was tested on 10 sputum specimens (8 Ziehl-positive specimens). Briefly, these specimens were inoculated in parallel with and without $2 \mathrm{mg} / \mathrm{L}$ rifampicin, under microaerophilic atmosphere and under normal atmosphere in the presence of acid ascorbic. Colony monitoring by fluorescence microscope was conducted every 12 hours for the first 48 hours and next every 8 hours. Microcolonies detected were identified by MALDI-TOF-MS

Statistical analysis. Comparisons of growth times were performed using Student's $t$ test to compare the average of a statistical sample to a fixed value. A $P$ value $<0.05$ was considered to be significant.

1. WHO Global Tuberculosis Report 2013. World Health Organization, Geneva (2013). http://www.who.int/tb/publications/global_report/en/. Accessed on 3rd December 2013.
2. Adler, H., Straub, C. \& Frei, R. Comparison of BacT/ALERT 3D, LowensteinJensen medium and Middlebrook 7H10/7H11 biplate for recovering mycobacteria from clinical specimens. Eur. J. Clin. Microbiol. 24, 499-500 (2005).

3. Samra, Z., Kaufman, L., Bechor, J. \& Bahar, J. Comparative study of three culture systems for optimal recovery of mycobacteria from different clinical specimens. Eur. J. Clin. Microbiol. 19, 750-754 (2000).

4. Boehme, C. C. et al. Rapid molecular detection of tuberculosis and rifampin resistance. N. Engl. J. Med. 11, 1005-1015 (2010).

5. Singh, S., Eldin, C., Kowalczewska, M. \& Raoult, D. Axenic culture of fastidious and intracellular bacteria. Trends Microbiol. 21, 92-99 (2013).

6. Drancourt, M. \& Raoult, D. Cost-effectiveness of blood agar for isolation of mycobacteria. PLoS Negl. Trop. Dis. 1, e83 (2007)

7. Leung, E., Minion, J., Benedetti, A., Pai, M. \& Menzies, D. Microcolony culture techniques for tuberculosis diagnosis: a systematic review. Int. J. Tuberc. Lung Dis. 16, 16-23 (2012).

8. El Khéchine, A., Couderc, C., Flaudrops, C., Raoult, D. \& Drancourt, M. Matrixassisted laser desorption/ionization time-of-flight mass spectrometry identification of mycobacteria in routine clinical practice. PLoS ONE 6, e24720 (2011).

9. El Khéchine, A., Henry, M., Raoult, D. \& Drancourt, M. Detection of Mycobacterium tuberculosis complex organisms in the stools of patients with pulmonary tuberculosis. Microbiology 155, 2384-2389 (2009).

10. Singh, S. et al. Cell extract-containing medium for the culture of intracellular fastidious bacteria. J. Clin. Microbiol. 51, 2599-2607 (2013).

11. Vilchèze, C., Hartman, T., Weinrick, B. \& Jacobs, W. R. Jr. Mycobacterium tuberculosis is extraordinarily sensitive to killing by a vitamin C-induced Fenton reaction. Nat. Commun. 4, 1881 (2013).

12. Mba Medie, F., Ben Salah, I., Henrissat, B., Raoult, D. \& Drancourt, M. Mycobacterium tuberculosis complex mycobacteria as amoeba-resistant organisms. PLoS One 6, e20499 (2011).

13. Kaufmann, S. H. How can immunology contribute to the control of tuberculosis? Nat. Rev. Immunol. 1, 20-30 (2001).

14. Neyrolles, O. et al. Is adipose tissue a place for Mycobacterium tuberculosis persistence? PLoS One 1, e43 (2006).

15. Clanton, T. L., Hogan, M. C. \& Gladden, L. B. Regulation of cellular gas exchange, oxygen sensing, and metabolic control. Compr. Physiol. 3, 1135-1190 (2013).

16. Dedieu, L., Serveau-Avesque, C., Kremer, L. \& Canaan, S. Mycobacterial lipolytic enzymes: a gold mine for tuberculosis research. Biochimie 95, 66-73 (2013).

17. Topuz, K. et al. Effect of hyperbaric oxygen therapy on the duration of treatment of spinal tuberculosis. J. Clin. Neurosci. 16, 1572-1577 (2009).

18. Grant, S. S., Kaufmann, B. B., Chand, N. S., Haseley, N. \& Hung, D. T. Eradication of bacterial persisters with antibiotic-generated hydroxyl radicals. Proc. Natl. Acad. Sci. U S A. 109, 12147-12152 (2012).

19. Kempker, R. R., Vashakidze, S., Solomonia, N., Dzidzikashvili, N. \& Blumberg, H. M. Surgical treatment of drug-resistant tuberculosis. Lancet Infect. Dis. 12, 157-166 (2012)

20. Patiño, S. et al. Autofluorescence of mycobacteria as a tool for detection of Mycobacterium tuberculosis. J. Clin. Microbiol. 46, 3296-3302 (2008).

\section{Acknowledgments}

The authors would like to thank Pascal Weber for his help with microscopy studies and Marielle Bedotto-Buffet for her help with the molecular techniques.

\section{Author contributions}

R.G. performed the experiments, reviewed data and wrote the manuscript; D.R. designed the experimental plan, provided with reagents and tools, and wrote the manuscript; M.D. designed the experiments, reviewed data and wrote the manuscript.

\section{Additional information}

Supplementary information accompanies this paper at http://www.nature.com/ scientificreports

Competing financial interests: The three authors are co-inventors of a pending patent on the culture and subculture of M. tuberculosis mycobacteria using the herein described protocol and MOD4 medium, which is owned by Aix-Marseille University.

How to cite this article: Ghodbane, R., Raoult, D. \& Drancourt, M. Dramatic reduction of culture time of Mycobacterium tuberculosis. Sci. Rep. 4, 4236; DOI:10.1038/srep04236 (2014)

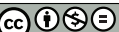

This work is licensed under a Creative Commons AttributionNonCommercial-NoDerivs 3.0 Unported license. To view a copy of this license, visit http://creativecommons.org/licenses/by-nc-nd/3.0 